\title{
Detection of Biogenic Magnetic Nanoparticles in Human Aortic Aneurysms
}

\author{
Y.A. Darmenko ${ }^{a, *}$, O.Yu. Gorobets ${ }^{a, b}$, S.V. Gorobets ${ }^{a}$, I. V. Sharay ${ }^{b}$ \\ AND O.M. LAZARENKO ${ }^{c}$ \\ ${ }^{a}$ National Technical University of Ukraine "Igor Sikorsky Kyiv Polytechnic Institute", \\ Peremogy Av. 37, 03056 Kyiv, Ukraine \\ ${ }^{b}$ Institute for Magnetism NAS of Ukraine, Vernadskiy Av. 36-b, 03142 Kyiv, Ukraine \\ ${ }^{c}$ State Scientific Institution "Scientific-Practical Centre of Preventive and Clinical Medicine", \\ Verchnya str., 5, Kiev, 01133, Ukraine
}

\begin{abstract}
The presence of biogenic magnetic nanoparticles (BMNs) in aortic aneurysm is detected using magnetic force microscopy. The presence of BMNs (single BMN and their chains) in aortic aneurysm may cause magneto-dipole interactions of these BMNs with BMNs of microorganisms - pathogens of heart and with artificial magnetic nanoparticles in the drug delivery systems. The accumulation of BMNs in the human heart can be attributed not only to the process of biomineralization BMNs directly in the tissues of heart but due to the accumulation of microorganisms - pathogens of heart that are natural producers of BMNs.
\end{abstract}

DOI: 10.12693/APhysPolA.133.738

PACS/topics: 87.64.-t, 87.19.Xx

\section{Introduction}

Magnetic nanoparticles of natural origin, i.e. biogenic magnetic nanoparticles (BMNs) are found in many organs of the human body. Normally BMNs are found in the heart, liver, spleen [1], adrenal glands [2], ethmoid bone [3] and brain [4]. BMNs number is much higher in inflammatory processes and pathologies, such as the formation of atherosclerotic plaques, the neurodegenerative (Alzheimer, Parkinson, Huntington [5]) and oncology than in norm [6].

The aim of this work is to determine the localization of BMN and their morphological characteristics in human heart tissues in pathologies caused by infectious diseases by means of magnetic force microscopy (MFM) and identification of potential producers of BMN among pathogenic and opportunistic microorganisms that cause heart diseases.

\section{Material and equipment}

The most common infectious disease of the heart is bacterial endocarditis. Infectious endocarditis can occur at any age. Men get sick 2 times more often than women [7].

The risk groups are: patients with valve prostheses, internal cardiac devices, congenital heart defects, chronic rheumatism, degenerative valve lesions. The risk of endocarditis also increases with hemodialysis, diabetes, human immunodeficiency virus [8].

*corresponding author; e-mail: darmenko.ea@ukr.net
During the disease, the valvular endocardium is affected, characterized by rapid development of valvular insufficiency, systemic complications and immunopathological manifestations. Infection occurs on the affected or pathologically altered valve [8].

Vegetations containing platelets, fibrin, erythrocytes, inflammatory cells and colonies of microorganisms are formed at endocarditis on the surface of the endocardium. Ulcers, ruptures, destruction of the valves, and the spread of infection to other structures of the heart, aneurysms may occur with the progression of the disease [8]. The development of infective endocarditis begins with the presence of bacteria or fungi in the blood and on the inner surface of the heart [9].

The causative agents of both infective endocarditis and a number of other diseases can be a large number of microorganisms. In $80 \%$ of cases, infectious endocarditis causes streptococci and staphylococci [8]. The most common causative agent of endocarditis is Staphylococcus aureus [7-9].

\section{Results and discussion}

The proteome of microorganisms was aligned with Magnetospirillum gryphiswaldense MSR-1 proteome using comparative genomics methods, the BMMs biomineralization mechanism in which has been studied in detail $[10,11]$. Most of the proteins that are involved in the biomineralization of BMNs in MTB are encoded in the magnetosome island (MAI) and is a manifestation of the genes of the magnetosome island $[11,12]$. The proteins MamA, MamB, MamM, MamE, MamO belong to proteins, without which the process of biomineralization of BMNs in MTB is impossible. Other proteins of the MTB MAI belong to regulatory proteins that are responsible 
for the control of shape, size, amount of BMNs in the cell, the formation of magnetosomal vesicles, and the formation of chains of BMNs [13]. MAI proteins MamJ and MamK are responsible for the formation of chains in the MTB.

Pairwise alignment methods were applied using the BLAST program of the National Center for Biotechnological Information for estimation of the degree of similarity between BMN biomineralization proteins of MTB Magnetospirillum gryphiswaldense MSR-1 and proteins of microorganisms. The following statistical criteria were taken into account for estimation of the degree of similarity of aligned sequences. Ident is the number of identical amino acid residues of proteins, compared, with optimal alignment. E-number is the number reflecting the statistical significance of the alignment. A decrease of its value indicates a lower level of the occurrence of the randomness when the amino acid residues of the proteins coincide. Length is the length of the alignment. The alignment length should be greater than 100 amino acid residues and the function of the proteins should be examined. The results of the alignment of the proteins are given in Table I.

It is known that it is sufficient to have homologues MAI MTB proteins: MamA, MamB, MamM, MamE, MamO for the formation of intracellular microorganisms crystalline BMNs, and the proteome must have homologues proteins MAI MTB: MamM, MamB, MamE, MamO to form intracellular amorphous BMNs. Therefore, in Table I, the microorganisms under investigation are divided into the corresponding groups. In addition, Table I shows the protein MamK, which is responsible for the formation of actin filaments and provides the formation of chains in cells of living organisms, including humans.

In Table I, the values of the E-number and ident are highlighted in bold type for the proteins if their functions do not coincide with the functions of the corresponding proteins in the MTB, as well as the length of the aligned sequences that is less than 100 amino acid residues. Italic font of values of the E-number and ident correspond to proteins which, at the moment, are defined in the database as hypothetical with unknown functions.

Thus, according to the results of the conducted studies, it can be concluded that all the microorganisms under investigation are potential producers of BMN. In addition, such microorganisms as: Staphylococcus aureus, Streptococcus pneumoniae, Coxiella burnetii and Bacteroides fragilis, have homologues of proteins MamA, MamB, MamE, MamO, MamM, therefore they are potential producers of intracellular crystalline BMN. Since the genomes of the microorganisms Staphylococcus aureus, Bacteroides fragilis, Streptococcus pneumoniae, Pseudomonas aeruginosa and Streptococcus agalactiae are not fully sequenced, it is impossible to draw conclusions about the presence of the MamK protein in them, which is responsible for the formation of chains. But it is known that crystalline BMNs form chains in the cell even without the presence of the MamK protein or its homologues
TABLE I

Alignment of proteins of Magnetospirillum gryphiswaldense MSR-1 with proteins of bacteria of infectious endocarditis pathogens forming intracellular BMN.

\begin{tabular}{|c|c|c|c|c|c|c|}
\hline \multirow{3}{*}{$\begin{array}{l}\text { Bacterium, } \\
\text { caus. agent of } \\
\text { endocarditis }\end{array}$} & \multicolumn{6}{|c|}{ Magnetospirillum gryphiswaldense MSR-1 } \\
\hline & \multirow{2}{*}{\multicolumn{6}{|c|}{$\begin{array}{l}\text { E-number/ident. [\%]/length of the alignment } \\
\text { MamA }\end{array}$}} \\
\hline & & & & & & \\
\hline \multicolumn{7}{|c|}{ Microorganisms that form intracellular crystalline BMN } \\
\hline \multirow{3}{*}{$\begin{array}{l}\text { S. aureus } \\
\text { C0673 }\end{array}$} & $2 \mathrm{e}-06$ & $4 \mathrm{e}-24$ & $7 \mathrm{e}-38$ & $1 \mathrm{e}-11$ & $8 \mathrm{e}-27$ & 7.1 \\
\hline & $25 \%$ & $26 \%$ & $30 \%$ & $29 \%$ & $42 \%$ & $29 \%$ \\
\hline & 126 & 274 & 257 & 274 & 177 & 93 \\
\hline \multirow{3}{*}{$\begin{array}{l}\text { B. fragilis } \\
\text { str. S36L5 }\end{array}$} & $4 \mathrm{e}-09$ & $6 \mathrm{e}-36$ & $4 \mathrm{e}-30$ & $5 \mathrm{e}-13$ & $3 \mathrm{e}-33$ & 3.1 \\
\hline & $26 \%$ & $30 \%$ & $30 \%$ & $30 \%$ & $47 \%$ & $55 \%$ \\
\hline & 155 & 275 & 261 & 175 & 163 & 20 \\
\hline \multirow{3}{*}{$\begin{array}{l}\text { Str. pneum. } \\
\text { GA41410 }\end{array}$} & 0.018 & $5 \mathrm{e}-28$ & $6 \mathrm{e}-24$ & $7 \mathrm{e}-04$ & $2 \mathrm{e}-26$ & 0.062 \\
\hline & $25 \%$ & $29 \%$ & $24 \%$ & $24 \%$ & $40 \%$ & $45 \%$ \\
\hline & 109 & 262 & 261 & 159 & 174 & 331 \\
\hline \multirow{3}{*}{$\begin{array}{l}\text { C. burnetii } \\
\text { RSA } 493\end{array}$} & $1 e-07$ & $1 \mathrm{e}-37$ & $5 \mathrm{e}-34$ & $2 \mathrm{e}-06$ & $2 \mathrm{e}-32$ & 3.9 \\
\hline & $24 \%$ & $28 \%$ & $28 \%$ & $25 \%$ & $43 \%$ & $26 \%$ \\
\hline & 164 & 276 & 293 & 193 & 176 & 66 \\
\hline \multicolumn{7}{|c|}{ Microorganisms that form intracellular amorphous BMN } \\
\hline \multirow{3}{*}{$\begin{array}{l}\text { Str. intermed. } \\
\text { B196 }\end{array}$} & 0.14 & $3 \mathrm{e}-29$ & $6 \mathrm{e}-27$ & $8 \mathrm{e}-04$ & $4 \mathrm{e}-22$ & - \\
\hline & $48 \%$ & $30 \%$ & $28 \%$ & $23 \%$ & $41 \%$ & - \\
\hline & 139 & 273 & 256 & 169 & 172 & - \\
\hline \multirow{3}{*}{$\begin{array}{l}\text { P. aeruginosa } \\
\text { M18** }\end{array}$} & 0.037 & $6 e-12$ & $2 \mathrm{e}-11$ & $1 \mathrm{e}-07$ & $6 e-34$ & $7 \mathrm{e}-05$ \\
\hline & $32 \%$ & $23 \%$ & $25 \%$ & $24 \%$ & $40 \%$ & $22 \%$ \\
\hline & 81 & 263 & 281 & 184 & 197 & 305 \\
\hline \multirow{3}{*}{$\begin{array}{l}\text { Str. agalact. } \\
\text { LMG } 14747\end{array}$} & 0.15 & $4 \mathrm{e}-27$ & $9 \mathrm{e}-23$ & 0.02 & $7 \mathrm{e}-26$ & 0.028 \\
\hline & $25 \%$ & $26 \%$ & $26 \%$ & $26 \%$ & $39 \%$ & $27 \%$ \\
\hline & 120 & 271 & 264 & 170 & 185 & 103 \\
\hline
\end{tabular}

** The microorganisms in which BMN was experimentally found [14].

due to the forces of magnetic dipole-dipole interaction between the BMNs in cells.

Based on the results of the study (Table I), the bacterium Streptococcus intermedius forms intracellular amorphous BMN, since it lacks the homolog of the MamA protein. With regard to the microorganisms Pseudomonas aeruginosa and Streptococcus agalactia (Table I), it is impossible to conclude what kind of BMN the can form since the genome is not fully decoded. The presence of amorphous intracellular BMN in Pseudomonas aeruginosa was confirmed experimentally [14].

Fungi that cause endocarditis are also potential producers of BMN, but since the fungal genomes (Table II) have not been fully sequenced, it is impossible to conclude today BMN with what properties they can biomineralize.

As indicated above, after the majority of inflammatory processes and infectious diseases accompanied by ischemia, serious cardiac complications appear in the human body, in particular, damage to valves and aneurysms [8]. To study the presence of BMN, a mitral valve, an aortic aneurysm and an aneurysm of the human heart was selected. The human mitral valve is replaced 
TABLE II

Alignment of Magnetospirillum gryphiswaldense MSR-1 proteins with fungal proteins of infectious endocarditis pathogens.

\begin{tabular}{c|c|c|c|c|c|c}
\hline \hline \multirow{2}{*}{$\begin{array}{c}\text { Fungi, } \\
\text { endocarditis } \\
\text { pathogens }\end{array}$} & \multicolumn{4}{|c}{$\begin{array}{c}\text { Magnetospirillum gryphiswaldense MSR-1 } \\
\text { E-number/ident. [\%]/length of the alignment }\end{array}$} \\
\cline { 2 - 7 } & MamA & MamB & MamM & MamO & MamE & MamK \\
\hline \multirow{2}{*}{ Aspergillus } & 28 & $7 \mathrm{e}-17$ & $3 \mathrm{e}-15$ & $\mathbf{0 . 0 7}$ & $\mathbf{0 . 0 6}$ & 0.009 \\
fum. Af293 & 25 & $25 \%$ & $27 \%$ & $\mathbf{2 8 \%}$ & $\mathbf{5 7 \%}$ & $25 \%$ \\
& 106 & 302 & 303 & $\mathbf{6 0}$ & $\mathbf{2 1}$ & 174 \\
\hline \multirow{2}{*}{ Histoplasma } & $\mathbf{3 e - 0 7}$ & $\mathrm{e}-11$ & $9 \mathrm{e}-07$ & $\mathbf{0 . 5 7}$ & 0.12 & 0.010 \\
caps. G186AR & $\mathbf{1 9 \%}$ & $32 \%$ & $23 \%$ & $\mathbf{3 0 \%}$ & $31 \%$ & $25 \%$ \\
& $\mathbf{1 6 5}$ & 187 & 258 & $\mathbf{8 0}$ & 94 & 175
\end{tabular}

because of the inability to perform its functions, namely, the expansion of the valves and the loss of elasticity.

The study was conducted using methods atomic force (AFM) and magnetic force microscopy (MFM) (Fig. 1).

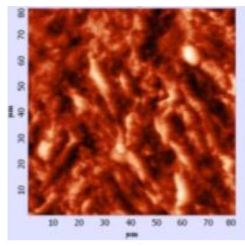

a1)

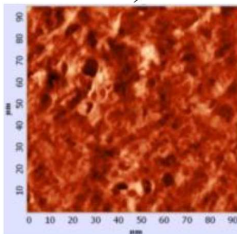

b1)

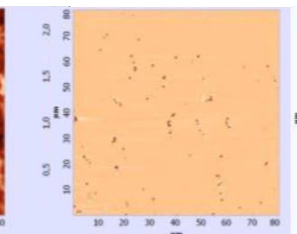

a2)

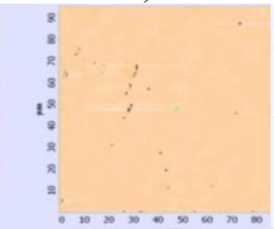

b2)

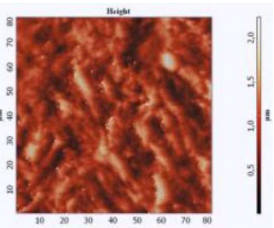

a3)

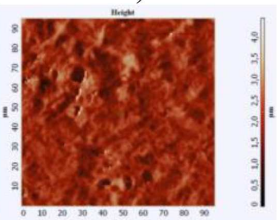

b3)
Fig. 1. Image aneurysm human heart: a1) - AFM image, a2) — MFM image, a3) — combined AFM and MFM image; image of aortic aneurysm: b1) AFM image, b2) MCM image, b3) — combined AFM and MFM image.

The magnetic probe MFM_LM series with chip size $3.4 \times 1.6 \times 0.3 \mathrm{~mm}$, coated by $\mathrm{CoCr}$ was used. This probe was used both for AFM and MFM imaging. The noncontact AFM (NC-AFM) mode was applied. The MFM scanning was carried out at constant distance from the sample surface after AFM scanning. The probe "lift" height was $100 \mathrm{~nm}$. The cantilever was calibrated using the test samples. Calibration of the probe was carried out immediately before the measurements. The figures 1 a3, b3 represent the overlapping of AFM topography image with MFM image. It means that MFM and AFM images were combines in one image (using Photoshop) with the purpose of revealing peculiarities of topography of the surface in the vicinity of BMNs. Figure 1 shows AFM and MFM images of pathologically altered areas of the human heart. Figure 1 shows that in the affected tissues of the human heart, both individual BMNs and their chains are formed [14]. It is known that the diameter of capillaries in the myocardium averages 5-6 microns, varies within $2.5-8$ microns $[15,16]$, and the average intercapillary distance is 15 microns (varies from 10 to 20 microns), which corresponds to the diameter and distance of dark areas on the AFM Image (Fig. 1). It can be assumed that the formation of individual BMNs, as well as their chains, occurs in the vicinity of the capillaries, which can lead, as already noted above, to the accumulation of microorganisms with BMN in the vicinity of the BMN heart tissue chains.

\section{Conclusion}

The paper identified as separate BMN and chains in the mitral valve in the heart aneurysm and aortic aneurysm humans. Also among the bacteria and fungi of cardiac pathogens, potential producers of BMN have been identified: Staphylococcus aureus C0673, Bacteroides fragilis str. S36L5, Streptococcus pneumoniae GA41410, Coxiella burnetii RSA 493, Streptococcus intermedius B196, Pseudomonas aeruginosa M18, Streptococcus agalactiae LMG 14747, Aspergillus fumigatus Af293, Histoplasma capsulatum G186AR.The magnetic dipole interaction of BMN in the human heart tissue and microorganisms and fungi with BMN can lead to the accumulation of magnetic nanoparticles in heart diseases. It must be taken into account when magnetically controlled targeted delivery of drugs in order to prevent toxic-allergic reactions and clogging of blood vessels.

\section{Acknowledgments}

This work was supported by the project has received funding from the European Union Horizon 2020 Research and Innovation Programme under Marie SkłodowskaCurie grant agreement No. 644348 (MagIC).

\section{References}

[1] P.P. Schultheiss-Grassi, F. Heller, J. Dobson, BioMetals. 10, 351 (1997).

[2] J.L. Kirschvink, J. Exper. Biol. 92, 333 (1981).

[3] R.R. Baker, J.G. Mather, J.H. Kennaugh, Nature. 301, 78 (1983).

[4] F. Brem, A.M. Hirt, M. Winklhofer, K. Frei, J. Res. Soc. Interface. 3, 833 (2006).

[5] D. Hautot, Q.A. Pankhurst, N. Khan, J. Dobson, Proc Biol Sci. 270, 62 (2003).

[6] A. Kobayashi, N. Yamamoto, J. Kirschvink, J. Jap. Soc. Powd. Powd. Metallurgy. 44, 94 (1997).

[7] A. Almas, M. Tariq, JCPSP. 18, 37 (2008).

[8] B. Hoen, X. Duval, N. Engl. J. Med. 368, 1425 (2013).

[9] D. Pierce, B.C. Calkins, K. Thornton, Am. Farm. Physician 85, 981 (2012).

[10] S. Ullrich, M. Kube, S. Schübbe, R. Reinhardt, J. Bacteriol. 187, 7176 (2005). 
[11] S. Schubbe, Ch.Wurdemann, J. Peplies, U. Heyen, C. Wawer, F. Glockner, D. Schuler, Appl. Environment. Microbiol. 72, 5757 (2006).

[12] M. Richter, M. Kube, D.A. Bazylinski, J. Bacteriol. 189, 4899 (2007).

[13] A. Lohße, PLoS ONE. 6, e25561 (2011).

[14] M. Vainshtein, N. Suzina, E. Kudryashova, E. Ariskina, Biol. Cell. 94, 29 (2002).
[15] L. Henquell, C.R. Microvasc Res. 12, 35 (1976).

[16] M. Steinhausen, H. Thederan, D. Nolinski, F.D. Dallenbach, A. Schwaier, Virchows Archiv A. 381, 13 (1978). 